\title{
SN-38-Loaded Polymeric Micelles NK012
}

National Cancer Institute

\section{Source}

National Cancer Institute. SN-38-Loaded Polymeric Micelles NK012. NCI Thesaurus.

Code C71521.

A formulation consisting of polymeric micelles loaded with the irinotecan metabolite SN-

38 with potential antineoplastic activity. SN-38-loaded polymeric micelles NK012 is an SN-

38-releasing nanodevice constructed by covalently attaching SN-38 to the block

copolymer PEG-PGlu, followed by self-assembly of amphiphilic block copolymers in an aqueous milieu. SN-38 (7-ethyl-10-hydroxy-camptothecin), a biological active metabolite of the prodrug irinotecan (CPT-11), binds to and inhibits topoisomerase I by stabilizing the cleavable complex between topoisomerase I and DNA, resulting in DNA breaks, inhibition of DNA replication, and apoptosis. SN-38 has been reported to exhibit up to 1,000 -fold more cytotoxic activity against various cancer cells in vitro than irinotecan. This formulation increases the water-solubility of SN-38 and allows the delivery of higher doses of SN-38 than those achievable with SN-38 alone. 